\title{
Unified description of ground and excited states of finite systems: The self-consistent $G W$ approach
}

\author{
F. Caruso, ${ }^{1,2, *}$ P. Rinke, ${ }^{1,2}$ X. Ren, ${ }^{1,2}$ M. Scheffler, ${ }^{1,2}$ and A. Rubio ${ }^{1,2,3}$ \\ ${ }^{1}$ Fritz-Haber-Institut der Max-Planck-Gesellschaft, Faradayweg 4-6, D-14195 Berlin, Germany \\ ${ }^{2}$ European Theoretical Spectroscopy Facility \\ ${ }^{3}$ Nano-Bio Spectroscopy Group and ETSF Scientific Development Centre, Universidad del País Vasco, CFM CSIC-UPVIEHU-MPC \\ and DIPC, Avenida Tolosa 72, E-20018 Donostia, Spain
}

(Received 13 December 2011; revised manuscript received 14 May 2012; published 14 August 2012)

\begin{abstract}
$G W$ calculations with a fully self-consistent Green's function $G$ and screened interaction $W$-based on the iterative solution of the Dyson equation-provide a consistent framework for the description of groundand excited-state properties of interacting many-body systems. We show that for closed-shell systems selfconsistent $G W$ reaches the same final Green's function regardless of the initial reference state. Self-consistency systematically improves ionization energies and total energies of closed-shell systems compared to $G_{0} W_{0}$ based on Hartree-Fock and (semi)local density-functional theory. These improvements also translate to the electron density, as exemplified by an improved description of dipole moments, and permit us to assess the quality of ground-state properties such as bond lengths and vibrational frequencies.
\end{abstract}

DOI: 10.1103/PhysRevB.86.081102

PACS number(s): 31.15.-p, 71.10.-w, 71.15.Qe

Many-body perturbation theory $(\mathrm{MBPT})^{1}$ in the $G W$ approximation of the electronic self-energy ${ }^{2,3}$ is presently the state-of-the-art method for describing the spectral properties of solids. ${ }^{4,5}$ Recently, it has steadily gained popularity for molecules and nanosystems. ${ }^{6}$ In addition, MBPT provides a prescription to extract total energies and structural properties from the $G W$ approximation and therefore is a consistent theoretical framework for single-particle spectra and total energies.

Due to its numerical cost and algorithmic difficulties, the $G W$ method has only recently been applied self-consistently (i.e., nonperturbatively) to atoms, ${ }^{7}$ molecules,${ }^{8}$ and molecular transport. ${ }^{6}$ Predominantly, $G W$ calculations are still performed perturbatively (one-shot $G_{0} W_{0}$ ) on a set of singleparticle orbitals and eigenvalues obtained from a preceding density-functional theory ${ }^{9}$ (DFT) or Hartree-Fock (HF) calculation. This procedure introduces a considerable starting-point dependence, ${ }^{10-12}$ which can be eliminated by iterating the Dyson equation to self-consistency. ${ }^{6-8,13}$ The resulting selfconsistent $G W$ (sc- $G W$ ) framework is a conserving approximation in the sense of Baym and Kadanoff ${ }^{14}$ (i.e., it satisfies momentum, energy, and particle number conservation laws). sc- $G W$ gives total energies ${ }^{15}$ free from the ambiguities of the $G_{0} W_{0}$ scheme, in which the results depend on the chosen total energy functional. ${ }^{7}$ However, as in any self-consistent theory, the question remains if the self-consistent solution of the Dyson equation is unique. This issue is fundamentally different from the initial-state dependence of $G_{0} W_{0}$. For HF (Ref. 16) and local-density approximation (LDA)/generalized gradient approximation $+U(\mathrm{GGA}+U)($ Ref. 17) calculations, it is well known that the self-consistency cycle can reach many local minima instead of the global minimum. Moreover, a previous sc- $G W$ study for the Be atom showed that normconserving pseudopotential calculations do not produce the same final $G W$ Green's function (and the corresponding ionization potential) as all-electron calculations. ${ }^{18}$

In this Rapid Communication, we demonstrate certain key aspects of the sc- $G W$ approximation for closed-shell molecules that make sc- $G W$ attractive as a general purpose electronic-structure method. First, the iteration of the Dyson equation produces a self-consistent Green's function that is independent of the starting point, and determines both the ground- and excited-state properties (quasiparticle spectra) of a given system on the same quantum-mechanical level. This distinguishes sc- $G W$ from other (partially) self-consistent $G W$ schemes, ${ }^{19,20}$ which do not lend themselves to totalenergy or ionic force calculations. Moreover, the uniqueness of the sc- $G W$ Green's function facilitates an unbiased assessment of the $G W$ approach, which was masked previously by the starting-point dependence of $G_{0} W_{0}$. Second, self-consistency improves total and quasiparticle energies compared to $G_{0} W_{0}$ based on HF or DFT in (semi)local approximations and yields good agreement with high level quantum-chemical calculations and photoemission data. Third, unlike $G_{0} W_{0}$, sc- $G W$ yields an associated ground-state electron density, whose quality is, e.g., reflected in the improved description of dipole moments. All these points taken together are essential for future developments in electronic-structure theory such as vertex functions and beyond $G W$ approaches.

In the $G W$ approximation the electron self-energy $\Sigma$ is defined in terms of the one-particle Green's function $G$ and the screened Coulomb interaction $W$ as

$$
\Sigma\left(\mathbf{r}, \mathbf{r}^{\prime}, \omega\right)=i \int \frac{d \omega^{\prime}}{2 \pi} G\left(\mathbf{r}, \mathbf{r}^{\prime}, \omega+\omega^{\prime}\right) W\left(\mathbf{r}, \mathbf{r}^{\prime}, \omega^{\prime}\right) e^{i \omega \eta},
$$

where $\eta$ is a positive infinitesimal, $W$ is the screened interaction, and spin variables are omitted for simplicity. More details about the calculation of $W$ are given in the Supplemental Material. $^{21}$ The Green's function can in turn be expressed in terms of the self-energy through the Dyson equation

$$
G^{-1}=G_{0}^{-1}-\left[\Sigma-v_{0}+\Delta v_{\mathrm{H}}\right],
$$

where $G_{0}$ refers to the Green's function of an independent particle system in an effective potential $v_{\text {eff. }} \Delta v_{\mathrm{H}}$ accounts for changes in the Hartree potential due to density differences between $G_{0}$ and $G$, and $v_{0}$ is the exact-exchange operator in HF or the Kohn-Sham exchange-correlation potential in DFT. 
The interdependence of Eqs. (1) and (2) is the very origin of the self-consistent nature of the $G W$ approach.

It is common practice, however, to solve Eqs. (1) and (2) just once, in the so-called one-shot $G_{0} W_{0}$ approximation. If Eq. (2) is not solved, the $G_{0} W_{0}$ quasiparticle energies are given in first-order perturbation theory as corrections to the reference eigenvalues $\left(\left\{\epsilon_{n}^{0}\right\}\right)^{22}$ as $\epsilon_{n}^{\mathrm{QP}}=\epsilon_{n}^{0}+\operatorname{Re}\left\langle\psi_{n}^{0}\right| \Sigma\left(\epsilon_{n}^{\mathrm{QP}}\right)-$ $v_{0}\left|\psi_{n}^{0}\right\rangle$.

In this work, Eqs. (1) and (2) were solved iteratively. Most importantly the screened interaction $W$ is also updated at each iteration, taking into account the full frequency dependence of the polarizability $\chi_{0}$ on the imaginary axis. In sc- $G W$ the excitation spectrum is given by the (integrated) spectral function:

$$
A(\omega)=-1 / \pi \int d \mathbf{r} \lim _{\mathbf{r}^{\prime} \rightarrow \mathbf{r}} \operatorname{Im} G\left(\mathbf{r}, \mathbf{r}^{\prime}, \omega\right) .
$$

The ground-state density $n(\mathbf{r})$ also follows directly from the Green's function: ${ }^{1}$

$$
n(\mathbf{r})=-2 i G\left(\mathbf{r}, \mathbf{r}, \tau=0^{-}\right) .
$$

The number of particles can be obtained through the integration of Eq. (4). This permits to verify the validity of the particle number conservation law at self-consistency (not shown for brevity) that is violated by non-self-consistent approaches as $G_{0} W_{0}$.

The situation is more complicated for the total energy. As alluded to above, for a given Green's function, different prescriptions exist to compute the associated total energy such as the Galitskii-Migdal formula, ${ }^{23}$ and the Luttinger-Ward ${ }^{24}$ or the Klein ${ }^{25}$ functionals. The latter two are variational in the sense that they are stationary at the self-consistent Green's function, and therefore might provide better total energies than the Galitskii-Migdal formula when evaluated with non-selfconsistent Green's functions. ${ }^{26,27}$ However, at self-consistency all three approaches are equivalent. Therefore, we choose the Galitskii-Migdal formula as it is easier to implement:

$$
E_{\mathrm{GM}}=-i \int \frac{d \omega}{2 \pi} \operatorname{Tr}\left\{\left[\omega+h_{0}\right] G(\omega)\right\}+E_{\mathrm{ion}},
$$

where $h_{0}$ is the one-particle term of the many-body Hamiltonian, i.e., the sum of the kinetic operator and the external potential. Equation (5) can be rewritten using the equation of motion for the Green's function ${ }^{21}$ as

$$
\begin{aligned}
E_{\mathrm{GM}}= & -i \sum_{i j} G_{i j}\left(\tau=0^{-}\right)\left[2 t_{j i}+2 v_{j i}^{\mathrm{ext}}+v_{j i}^{\mathrm{H}}+\Sigma_{j i}^{\mathrm{x}}\right] \\
& -i \sum_{i j} \int \frac{d \omega}{2 \pi} G_{i j}(\omega) \Sigma_{j i}^{\mathrm{c}}(\omega) e^{i \omega \eta}+E_{\mathrm{ion} .}
\end{aligned}
$$

Here $t$ denotes the kinetic-energy operator, $v^{\mathrm{H}}$ and $v^{\text {ext }}$ the Hartree and external potentials, and $\Sigma^{\mathrm{x}}$ and $\Sigma^{\mathrm{c}}$ are the exchange and correlation parts of the self-energy, respectively. In Eqs. (5) and (6) we suppressed spin variables, i.e., we assumed spin degeneracy. The trace of Eq. (5) is expressed as the sum over basis functions in Eq. (6) and the frequency integration is conveniently performed along the imaginary axis. $^{28}$

For comparison, we also computed $G_{0} W_{0}$ total energies with different starting points. However, as indicated above,
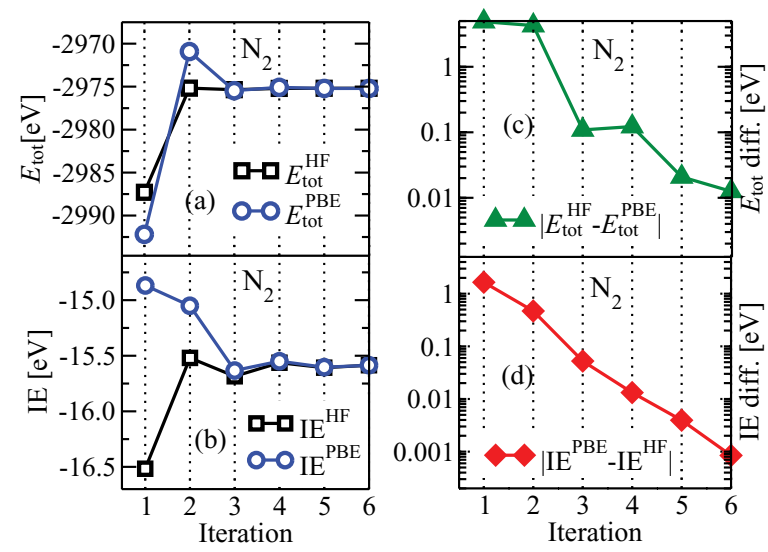

FIG. 1. (Color online) Total energy (a) and ionization energy (b) of $\mathrm{N}_{2}$ at each iteration of the sc- $G W$ loop for a HF and PBE input Green's function, for the augmented correlation-consistent polarized valence quadrupole zeta (aug-cc-pVQZ) (Ref. 34) basis set. The (absolute values of the) differences arising from HF and PBE initializations vanish exponentially for both total energy (c) and ionization energy (d).

$G_{0} W_{0}$ total energies are not uniquely defined, because the Green's function and the self-energy are never on the same level. If, for example, the Dyson equation is not solved, $G_{0}$ and $\Sigma_{0}=G_{0} W_{0}$ enter Eq. (6). If the Dyson equation is solved, the resulting $G_{1}$ is still inconsistent with $\Sigma_{0}$. In the following we refer to the combination of $G_{0}$ and $\Sigma_{0}$ in Eq. (6) as $G_{0} W_{0}$ total energy and denote the corresponding starting point with @ starting point.

We have implemented sc- $G W$ in the all-electron electronicstructure code FHI-AIMS. ${ }^{29,30}$ Equations (1)-(4) and (6) are solved in a numerical atomic orbital (NAO) basis using the resolution of identity technique to treat all two-particle operators efficiently. ${ }^{30,31}$ All calculations are performed on the imaginary frequency axis and the spectral function is obtained by analytic continuation to the real frequency axis. ${ }^{30}$ The analytic continuation constitutes the only approximation of our implementation of the sc- $G W$ method. Further details of the implementation will be given elsewhere. ${ }^{32}$

In Fig. 1 we demonstrate for $\mathrm{N}_{2}$ that the sc- $G W$ Green function provides total energies [Fig. 1(a)] and vertical ionization energies [Fig. 1(b)] that are independent of the starting point. Figure 1 explicitly illustrates this point, starting the selfconsistency cycle with HF and DFT in the Perdew, Burke, and Ernzerhof $(\mathrm{PBE})^{33}$ generalized gradient approximation, but other initializations such as the local-density approximation (LDA) or the simple Hartree approximation produce the same final sc- $G W$ Green's function (not shown). The deviation in the Green's function exemplified by the (absolute value of the) total energy difference [Fig. 1(c)] and the ionization energy difference [Fig. 1(d)] converges exponentially fast with the number of iterations, canceling the starting-point dependence. Further tests performed on a set of 30 closedshell molecules (see Fig. 4 and the Supplemental Material ${ }^{21}$ ) confirm this fact and demonstrate that sc- $G W$ provides a recipe for linking different reference systems of independent electrons (or noninteracting Kohn-Sham particles) to a unified interacting many-body state. 


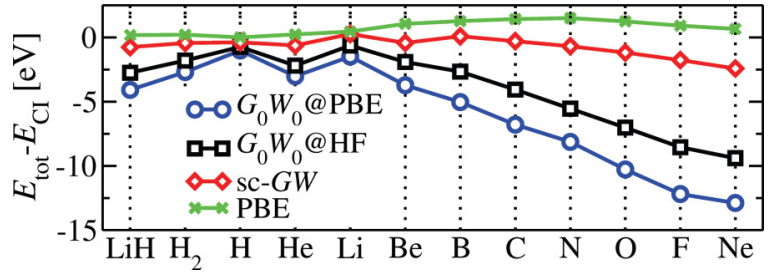

FIG. 2. (Color online) Difference between Galitskii-Migdal total energies $\left(E_{\mathrm{GM}}\right)$ and full configuration interaction values $\left(E_{\mathrm{CI}}\right)$ (Refs. 35-37), with $E_{\mathrm{GM}}$ evaluated from sc- $G W, G_{0} W_{0} @ \mathrm{HF}$, and $G_{0} W_{0} @$ PBE. PBE total energies are included for comparison. The calculations were performed using the augmented correlationconsistent polarized valence five zeta (aug-cc-pV5Z) basis set. (Ref. 34)

Having established the important point that the sc- $G W$ solution is independent of the starting point for the set of closed-shell molecules studied here, we now turn to an assessment of the performance of the $G W$ approach for ionization potentials, electron densities, and total energies. For elements in the first two rows of the Periodic Table (i.e., $Z=1-10)$ and small molecules such as $\mathrm{H}_{2}$ and $\mathrm{LiH}$, accurate reference data from configuration interaction $(\mathrm{CI})$ calculations are available. ${ }^{35-37}$ Figure 2 reports the difference to $\mathrm{CI}$ values for basis set converged sc- $G W, G_{0} W_{0} @ \mathrm{PBE}$, and $G_{0} W_{0} @ \mathrm{HF}$ calculations. A subset of these has previously been calculated using sc- $G W,{ }^{7}$ and our results are in excellent agreement with the published results. In line with previous calculations for the electron gas, ${ }^{26,28,38}$ atoms, and small molecules, ${ }^{7} G_{0} W_{0}$ total energies (in various flavors) tend to be too negative. The self-consistent treatment largely (but not fully) corrects this overestimation and provides total energies in more satisfying agreement with full CI. The remaining overestimation provides a clear and unbiased quantification of the required vertex corrections in a beyond $G W$ treatment.

For practical purposes, total energy differences are far more important than absolute total energies. However, for sc- $G W$ only one study has reported ground-state properties and found that sc- $G W$ gives lattice constants of $\mathrm{Si}$ and $\mathrm{Na}$ in good agreement with experiments. To assess ground-state properties, such as the equilibrium atomic structure, would in principle require atomic forces (i.e., derivatives of the total energy with respect to atomic coordinates), which are still needed for sc- $G W$. For diatomic molecules, however,
TABLE I. Equilibrium bond length $d$, vibrational frequency $v_{\text {vib }}$, dipole moment $\mu$, and binding energy $E_{\mathrm{b}}$ of the $\mathrm{CO}$ dimer. Units are, respectively, $\AA \mathrm{cm}^{-1}, \mathrm{D}$, and $\mathrm{eV}$. All calculations were performed with a Tier 4 basis set.

\begin{tabular}{lcccc}
\hline \hline $\mathrm{CO}$ & $d$ & $\nu_{\text {vib }}$ & $\mu$ & $E_{\mathrm{b}}$ \\
\hline Expt. (Ref. 39) & 1.128 & 2169 & 0.11 & 11.11 \\
sc- $G W$ & 1.118 & 2322 & 0.07 & 10.19 \\
$G_{0} W_{0} @ \mathrm{HF}$ & 1.119 & 2647 & & 11.88 \\
$G_{0} W_{0} @ \mathrm{PBE}$ & 1.143 & 2322 & & 12.16 \\
(EX+cRPA)@HF & 1.116 & 2321 & & 10.19 \\
(EX+cRPA)@PBE & 1.137 & 2115 & & 10.45 \\
PBE & 1.135 & 2128 & 0.20 & 11.67 \\
$\mathrm{HF}$ & 1.102 & 2448 & -0.13 & 7.63 \\
\hline \hline
\end{tabular}

structural properties such as vibrational frequencies, bond lengths, and binding energies can be determined directly from the potential energy curve. Other ground-state properties, e.g., dipole moments, can be inferred directly from the electron density. For brevity, we only present the case of $\mathrm{CO}$ here and refer to a future publication for a more detailed discussion of ground-state properties in sc- $G W .{ }^{32}$

In Table I we report the experimental values for the bond length $d$, vibrational frequency $v_{\text {vib }}$, dipole moment $\mu$, and binding energy $E_{\mathrm{b}}$ of $\mathrm{CO}$ (Ref. 39) together with the theoretical values obtained from several perturbative and nonperturbative approaches. DFT in the exact-exchange plus correlation in the random-phase approximation (EX + cRPA) based on PBE is remarkably accurate for the bond length and vibrational frequency of CO. ${ }^{40}$ However, as $G_{0} W_{0}, \mathrm{EX}+\mathrm{cRPA}$ exhibits a considerable starting-point dependence and gives no direct access to dipole moments. In sc- $G W$, the quality of the new density, obtained through Eq. (4), is manifested in the improved dipole moment, which is in much better agreement with the experimental value than in PBE and HF. Additional information on the quality of the sc- $G W$ electron density is reported in the Supplemental Material. ${ }^{21}$ The vibrational frequency, on the other hand, is overestimated and not substantially different from the perturbative $G_{0} W_{0}$ values. Self-consistency overcorrects the overestimation of the $G_{0} W_{0}$ binding energy, resulting in an underestimation of about $1 \mathrm{eV}$ for $E_{\mathrm{b}}$ compared to experiment. Similarly, the sc- $G W$ bond length is slightly too small and is close to $G_{0} W_{0} @$ HF. This assessment of the $G W$ approach for ground-state properties,

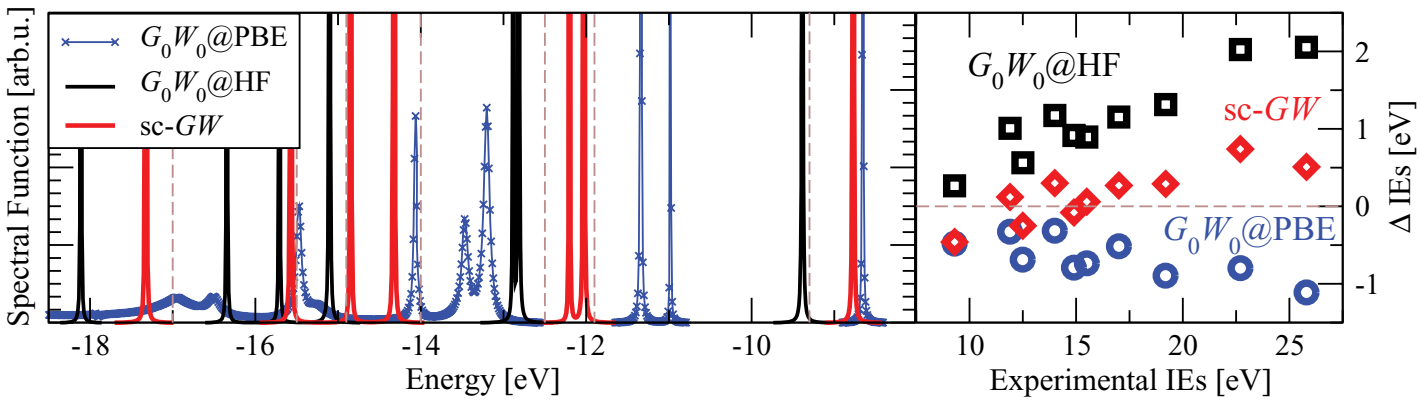

FIG. 3. (Color online) Left: The spectral function of benzene calculated with a Tier 2 basis set. Vertical dashed lines are located at experimental vertical ionization energies from Ref. 46. Right: Comparison of experimental (Ref. 46) and theoretical vertical ionization energies (VIEs) extracted from the spectral function of benzene for sc- $G W, G_{0} W_{0} @ \mathrm{HF}$, and $G_{0} W_{0} @ \mathrm{PBE}$. 


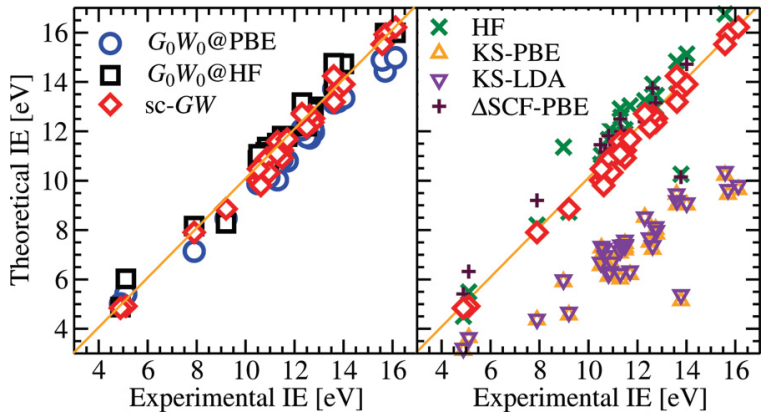

FIG. 4. (Color online) First vertical ionization energy (VIE) for 30 closed-shell molecules composed of two to eight atoms. Experimental values are taken from Ref. 39. Results from PBE total energy differences ( $\triangle \mathrm{SCF}-\mathrm{PBE})$ are included for comparison.

facilitated by sc- $G W$, clearly indicates where future challenges in going beyond $G W$ lie.

Finally, we turn to the description of spectral properties. For the homogeneous electron gas (HEG), Holm and von Barth first reported a deterioration of the spectral properties ${ }^{13}$ in sc- $G W$ compared to $G_{0} W_{0} @$ LDA. For the spectra of simple solids such as silicon and sodium, controversy then arose, with some authors advocating self-consistency ${ }^{41}$ and others dismissing it. ${ }^{38,42}$ Part of this controversy can be traced back to convergence difficulties in the early all-electron calculations, ${ }^{43}$ while the influence of the pseudopotential approximation in $G W$ turned out to be larger than initially anticipated. ${ }^{44}$

To test the quality of the sc- $G W$ spectra we chose the benzene molecule as a benchmark, for which the sc- $G W$ spectral function in Fig. 3 is compared to the $G_{0} W_{0} @ \mathrm{HF}$ and $G_{0} W_{0} @ \mathrm{PBE}$ ones calculated using Eq. (3). The vertical ionization energies (VIEs) shown in the right panel of Fig. 3 correspond to the peak positions in the spectral function. All the peaks reported in the left panel of Fig. 3 correspond to occupied quasiparticle states and the associated energy can be directly related to ionization energies as measured in photoemission spectroscopy. The $G_{0} W_{0}$ quasiparticle energies-reported in the right panel of Fig. 3-depend strongly on the starting point: HF-(PBE-)based $G_{0} W_{0}$ has a tendency to overestimate (underestimate) VIEs. The deviation between $G_{0} W_{0} @ \mathrm{HF}$ and $G_{0} W_{0} @ \mathrm{PBE}$ is $\simeq 0.5 \mathrm{eV}$ for the first ionization energy and can be as large as $\simeq 3 \mathrm{eV}$ for lower lying quasiparticle states. Furthermore, due to overscreening $G_{0} W_{0} @ \mathrm{PBE}$ yields a large broadening (i.e., short lifetimes) for quasiparticle peaks below $-12 \mathrm{eV}$. We emphasize that those peaks are not plasmon satellites, but quasiparticle states with a short lifetime. The short lifetime arises from the small highest occupied molecular orbital (HOMO)-lowest unoccupied molecular orbital (LUMO) gap in PBE that allows quasiparticle states to decay through the creation of electron-hole pairs. ${ }^{45}$ At selfconsistency, the quasiparticle energies are uniquely defined, the systematic (over)underestimation of $G_{0} W_{0}$ calculations is considerably reduced, and the resulting quasiparticle energies are in better agreement with photoemission data. ${ }^{46}$

We further assessed the quality of sc- $G W$ quasiparticle energies for the set of 30 molecules calculated by Rostgaard et al. ${ }^{8}$ For brevity our results are summarized in Fig. 4 and we refer to the Supplemental Material for the actual numerical values. $^{21}$ In Ref. 8 sc- $G W$ was based on the frozen-core approximation, whereas in our work core electrons were also treated fully self-consistently. Core-valence coupling is therefore included in our implementation and is likely responsible for the deviation of $0.1-0.5 \mathrm{eV}$ in the first VIEs between our and Rostgaard et al.'s sc- $G W$ calculations. As for benzene, $G_{0} W_{0} @ \mathrm{HF}$ tends to overestimate VIEs, while $G_{0} W_{0} @ \mathrm{PBE}$ underestimates. sc- $G W$ also slightly underestimates the VIEs, but gives an average deviation of only $2 \%$ compared to $6 \%$ in $G_{0} W_{0} @ \mathrm{PBE}$ and $4 \%$ in $G_{0} W_{0} @ \mathrm{HF} . \mathrm{PBE}$ and HF present two extreme starting points. In PBE the gap between the HOMO and the LUMO is severely underestimated, while in $\mathrm{HF}$ it is considerably overestimated. This in part explains the behavior of $G_{0} W_{0} @ \mathrm{PBE}$ and $G_{0} W_{0} @ \mathrm{HF}$. Since the screening strength is inversely proportional to the HOMO-LUMO gap, $G_{0} W_{0} @ \mathrm{PBE}$ overscreens and $G_{0} W_{0} @ \mathrm{HF}$ underscreens. By tuning the fraction of exact exchange in the ground states, as, e.g., in hybrid functionals, the deviation between $G_{0} W_{0}$ and experiment could be further reduced for this data set. However, this procedure is neither predictive, nor universal or transferable, because different systems will require a different amount of exact exchange. To really assess the quality of the $G W$ approximation, self-consistency is therefore indispensable. From this we conclude that sc- $G W$ systematically improves the spectral properties of the systems considered here as compared to perturbative $G W$. More work is needed to investigate the quality of sc- $G W$ for a wider range of systems and materials, including transition metals or rare earth elements where correlations are stronger.

In summary, we have demonstrated that sc- $G W$ is independent of the starting point for closed-shell molecules. Selfconsistency improves the total energy and the spectral properties of the test sets compared to $G_{0} W_{0}$ based on HF or PBE, whereas structural properties worsen compared to EX + cRPA. Moreover, the sc- $G W$ electron densities improve the description of the dipole moment of CO. The sc- $G W$ approach therefore provides a unified theory for the electronic groundand excited-state properties of many-body systems. Most importantly, sc- $G W$ gives unambiguous reference data that is essential for developing vertex corrections for (bio)molecules, nanostructures, and extended systems, in particular, for the challenging class of "strongly correlated" materials.

A.R. acknowledges financial support from MEC (FIS201165702-C02-01), Grupos Consolidados UPV/EHU del Gobierno Vasco (IT-319-07), and the European Research Council (ERC-2010-AdG -No. 267374).

\footnotetext{
caruso@fhi-berlin.mpg.de

${ }^{1}$ A. L. Fetter and J. D. Walecka, Quantum Theory of Many-Particle Systems (Dover, New York, 2003).
}

${ }^{2}$ L. Hedin, Phys. Rev. 139, A796 (1965).

${ }^{3}$ F. Aryasetiawan and O. Gunnarsson, Rep. Prog. Phys. 61, 237 (1998). 
${ }^{4}$ W. G. Aulbur, L. Jönsson, and J. W. Wilkins, Solid State Phys. 54, 1 (2000)

${ }^{5}$ G. Onida, L. Reining, and A. Rubio, Rev. Mod. Phys. 74, 601 (2002).

${ }^{6}$ K. S. Thygesen and A. Rubio, Phys. Rev. B 77, 115333 (2008).

${ }^{7}$ A. Stan, N. E. Dahlen, and R. van Leeuwen, J. Chem. Phys. 130, 114105 (2009).

${ }^{8}$ C. Rostgaard, K. W. Jacobsen, and K. S. Thygesen, Phys. Rev. B 81, 085103 (2010).

${ }^{9}$ W. Kohn and L. J. Sham, Phys. Rev. 140, A1133 (1965).

${ }^{10}$ P. Rinke, A. Qteish, J. Neugebauer, C. Freysoldt, and M. Scheffler, New J. Phys. 7, 126 (2005).

${ }^{11}$ F. Fuchs, J. Furthmüller, F. Bechstedt, M. Shishkin, and G. Kresse, Phys. Rev. B 76, 115109 (2007).

${ }^{12}$ P. Rinke, A. Qteish, J. Neugebauer, and M. Scheffler, Phys. Status Solidi B 245, 929 (2008).

${ }^{13}$ B. Holm and U. von Barth, Phys. Rev. B 57, 2108 (1998).

${ }^{14}$ G. Baym and L. P. Kadanoff, Phys. Rev. 124, 287 (1961).

${ }^{15}$ C.-O. Almbladh, U. V. Barth, and R. V. Leeuwen, Int. J. Mod. Phys. B 13, 535 (1999).

${ }^{16}$ A. J. W. Thom and M. Head-Gordon, Phys. Rev. Lett. 101, 193001 (2008).

${ }^{17}$ B. Meredig, A. Thompson, H. A. Hansen, C. Wolverton, and A. van de Walle, Phys. Rev. B 82, 195128 (2010).

${ }^{18}$ K. Delaney, P. García-González, A. Rubio, P. Rinke, and R. W. Godby, Phys. Rev. Lett. 93, 249701 (2004).

${ }^{19}$ S. V. Faleev, M. van Schilfgaarde, and T. Kotani, Phys. Rev. Lett. 93, 126406 (2004).

${ }^{20}$ M. Shishkin and G. Kresse, Phys. Rev. B 75, 235102 (2007).

${ }^{21}$ See Supplemental Material at http://link.aps.org/supplemental/ 10.1103/PhysRevB.86.081102 for the numerical values of the data reported in Fig. 4, details on the computation of $W$ and the derivation of Eq. (6).

${ }^{22}$ M. S. Hybertsen and S. G. Louie, Phys. Rev. B 34, 5390 (1986).

${ }^{23}$ V. Galitskii and A. Migdal, Sov. Phys. JETP 7, 96 (1958).

${ }^{24}$ J. M. Luttinger and J. C. Ward, Phys. Rev. 118, 1417 (1960).
${ }^{25}$ A. Klein, Phys. Rev. 121, 950 (1961).

${ }^{26}$ B. Holm, Phys. Rev. Lett. 83, 788 (1999).

${ }^{27}$ N. E. Dahlen and R. van Leeuwen, J. Chem. Phys. 122, 164102 (2005).

${ }^{28}$ P. García-González and R. W. Godby, Phys. Rev. B 63, 075112 (2001).

${ }^{29}$ V. Blum, R. Gehrke, F. Hanke, P. Havu, V. Havu, X. Ren, K. Reuter, and M. Scheffler, Comput. Phys. Commun. 180, 2175 (2009).

${ }^{30} \mathrm{X}$. Ren, P. Rinke, V. Blum, J. Wieferink, A. Tkatchenko, A. Sanfilippo, K. Reuter, and M. Scheffler, New J. Phys. 14, 053020 (2012).

${ }^{31}$ O. Vahtras, J. Almlöf, and M. Feyereisen, Chem. Phys. Lett. 213, 514 (1993).

${ }^{32}$ F. Caruso, X. Ren, P. Rinke, A. Rubio, and M. Scheffler (unpublished).

${ }^{33}$ J. P. Perdew, K. Burke, and M. Ernzerhof, Phys. Rev. Lett. 77, 3865 (1996).

${ }^{34}$ T. H. Dunning, J. Chem. Phys. 90, 1007 (1989).

${ }^{35}$ S. J. Chakravorty, S. R. Gwaltney, E. R. Davidson, F. A. Parpia, and C. F. Fischer, Phys. Rev. A 47, 3649 (1993).

${ }^{36}$ X. Li and J. Paldus, J. Chem. Phys. 118, 2470 (2003).

${ }^{37}$ L. Wolniewicz, J. Chem. Phys. 99, 1851 (1993).

${ }^{38}$ A. Kutepov, S. Y. Savrasov, and G. Kotliar, Phys. Rev. B 80, 041103 (2009).

${ }^{39} \mathrm{http} / / / \mathrm{cccbdb}$. nist.gov/.

${ }^{40}$ X. Ren, P. Rinke, and M. Scheffler, Phys. Rev. B 80, 045402 (2009).

${ }^{41}$ W. Ku and A. G. Eguiluz, Phys. Rev. Lett. 89, 126401 (2002).

${ }^{42}$ W.-D. Schöne and A. G. Eguiluz, Phys. Rev. Lett. 81, 1662 (1998),

${ }^{43}$ C. Friedrich, A. Schindlmayr, S. Blügel, and T. Kotani, Phys. Rev. B 74, 045104 (2006).

${ }^{44}$ R. Gomez-Abal, X. Li, M. Scheffler, and C. Ambrosch-Draxl, Phys. Rev. Lett. 101, 106404 (2008).

${ }^{45}$ A. Marini and A. Rubio, Phys. Rev. B 70, 081103 (2004).

${ }^{46}$ C. Fridh, L. Asbrink, and E. Lindholm, Chem. Phys. Lett. 15, 282 (1972). 